\title{
Sordarin - the antifungal antibiotic with unique modus operandi
}

\author{
Yutian Shao ${ }^{1}$, Eliza Molestak ${ }^{2}$, Weike $\mathrm{Su}^{1}$, Marek Stankevič² ${ }^{2}$, and Marek Tchorzewski² \\ ${ }^{1}$ Collaborative Innovation Center of Yangtze River Delta Region Green Pharmaceuticals, \\ Zhejiang University of Technology, Hangzhou \\ ${ }^{2}$ Maria Curie-Sklodowska University
}

July 2, 2021

\begin{abstract}
Fungal infections cause serious problems in many aspects of human life; especially infections by fungal species represent problems in immunocompromised patients. Current antifungal antibiotics target various metabolic pathways, predominantly the cell wall or cellular membrane. However, numerous compounds are available to combat fungal infections, their efficacy is far from being satisfactory and some of them display substantial toxicity. The emerging resistance represents a serious issue as well; thus, there is a considerable need for new anti-fungal compounds with lower toxicity and higher effectiveness. One of the unique antifungal antibiotics is sordarin, the only known compound that acts on the fungal translational machinery per se. It has been shown that sordarin inhibits protein synthesis at the elongation step of the translational cycle, acting on eukaryotic elongation-factor-2. In this review, we are aiming to deliver a robust scientific platform promoting the development of antifungal compounds, especially focusing on molecular action of sordarin.
\end{abstract}

\section{Hosted file}

Manuscript figures and tables.pdf available at https://authorea.com/users/423278/articles/ 528778-sordarin-the-antifungal-antibiotic-with-unique-modus-operandi 